\title{
Design of Home Circulation: Application to Smart Homes
}

\author{
Sabah Al-Fedaghi \\ Computer Engineering Department, Kuwait University \\ sabah.alfedaghi@ku.edu.kw
}

\begin{abstract}
This paper contributes to the notion of "house as machine" by proposing a methodology for producing engineering-type diagrams of machine processes that can be applied to smart home design in resolving such issues as positions of sensors, types of sensors, activity classifications, and activity recognition. The diagrammatic methodology is based on the conceptual model of space as a synchronic order of states (stages): creation, release, transfer, receive, and process. It views space in terms of dynamic content with stages and connections (flows) to create a representation of the flows in a home. The resultant depiction seems suitable for use in dèvigning of smart homes.
\end{abstract}

Keywords: Conceptual representations, hous as machine smant home, sensors, activity recognition, inferences

\section{Introduction}

According to Le Corbusier [1], a-house is a machine for living in. It is also claimed that "The city as a machine Sunctions according to the laws of a kind of urban physics" [2]. This notion has a profound impact on "architecture as architecture":

The practice of architecture as architecture moved towards a social engineering practice. The cental proposition of architecture as social engineering is that specific social outcomes can be engineered by manipulating architecture this way and that. In other words the relation between form and function in architecture is analogous to similar problems dealt with by engineers. If architecture is indeed social engineering then it needs a theory toxplain how it works. The paradigm of the machine filled this need. [3] (italic added)

A machine, inchis sense, indicates a system of activities and actions:

We use a machine, or the drawing of a machine, to symbolize a particular action of the machine. For instance, we give someone such a drawing and assume that he will derive the movement of the parts from it. [4]

This notion of machine is a well-established concept in science and philosophy. In philosophy, drawing on Deleuze and Guattari [5], Bryant [6] declares that "all objects" can be understood as machines:

A machine is something through which matters flow, undergoing a series of transformations as a result of the operations within the machine, producing a particular output. If, for example, we take a machine such as a tree, this is a machine through which flows of sunlight, water, carbon dioxide, minerals in the soil, etc., flow. Through a series of operations, the machine transforms those flows of matter, those other machines that pass through it, into various sorts of cells such as photosynthetic cells and bark, while also producing oxygen. [6] 
A machine here refers to a "theater" of representation of a pattern of events (even in the setting of a space), not a production factory [5]. Thus, the schema of a house is the screenplay of a composition played over a physical space.

This paper contributes to the notion of house as machine by proposing a methodology for producing engineering-like schemata to diagram the working of machines, thus facilitating a resolution to such issues in smart home design as position of sensors, activity classifications, and activity recognition. A kitchen provides the script for a machine that transfers, receives, and processes things, resulting in creation of other things that are released and transferred outside the kitchen. A home air-conditioning system is a machine by which hot air is transferred, received, and released to create cold air that is transferred and received by every unit of space in the house. In such a conceptualization, the house is a machine constituted of submachines by which people, food, air, water, electricity, pets, ... are processed, created, released, transferred, and received contiguously. Each of them has its own streams of flows and stages of handling with minimum congestion or stoppage. The process of shifting from such a blueprint to a realized home creates a functioning machine with parts assembled fôr the ultimate purpose of "creating" a confined environment.

This methodology for diagramming machinesutilizes a diagrammatic language called the Flowthing Model (FM), a modeling technique that has been used in several applications (e.g., [7-11]). For the sake of a complete presentation, the basic notions in FM are briefly described in the next section. The example of a tree as a machine is a new contribution. FM is applied to smart homes in the remaining sections as follows:

- Section 3 examines positioning of sensôns in accordance with architectural diagrams. Such positioning treats each internal flow like a black box and thus does not distinguish degree of progress within a given stage.

- Section 4 discusses construction of an ontology to classify and analyze activities. The papepresents an alternative pure conceptualization of activities that does not involve such hardware notions as sensors.

- Section 5 briefly discusses the exploitation of logical semantic reasoning for activity recognition.

\section{Flowthing Mode}

The Flowthing Model (FM) was inspired by the many types of flows that exist in diverse fields. including information flows, signal flows, and data flows in communication models. This model is a diagrammatic schema that uses flowthings to representa range of items, for example, electrical, mechanical, chemical, and thermal signals, circulating blood, food, concepts, pieces of data, and so on. Flow things are defined as what can be created, released, transferred, processed, and received (see Figure 1). Hereafter, flowthings are referred to as things. Note that what we call a thing is not necessarily a substance in the philosophical sense; e.g., heat is a thing that is created, released, transferred, received, and processed.

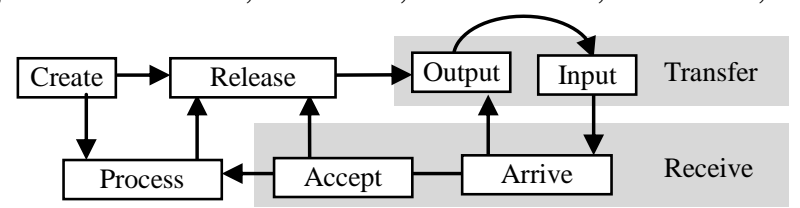

Figure 1. Flow Machine 
FM depicts processes using flow machines that are formed by up to six stages (states) occurring in a flow. Hereafter, flow machines will be referred to as machines. Machines form the organizational structure of whatever is described; in our study, these are humans and their physical and nonphysical processes. These processes can be embedded in a network of assemblies called spheres in which the processes of flow machines take place.

Starting in the lower right corner of Figure 1, the stages occurring in a flow machine can be described as follows:

Arrive: A thing reaches a new machine.

Accepted: A thing is permitted to enter a machine. If arriving things are always accepted, Arrive and Accept can be combined as a Received stage.

Processed (changed): The thing goes through some kind of transformation that changes it without creating a new thing.

Released: A thing is marked as ready to be transferred outside the machine.

Transferred: The thing is transported somewhere from/to outside the machine.

Created: A new thing is born (created) in a machine.

The machine shown in Figure 1 is a generalization of the typical machine model of input-process-output used in many scientific and engineering fields. In general, a machine is thought to be an abstract machine that recejves processes, creates, releases, and transfers things. The stages in this machine are mutually exclusive (i.e., a thing in the Process stage cannot be simultaneously in the Create stage or the Release stage at the same time). An additional stage of Storage can also be added to any machine to represent the storage of things, howeverCstorage is not an exclusive stage because there can be stored process things, stored created things, etc.

FM also uses the notions of spheres and subspheres. These are the network environments and relationships of machines and submachines. Multiple machines can exist in a sphere if needed. Asphere can be a person, an organ, an entity (e.g., a company, a customer), a location (a laboratory, a waiting room, a house), a communication medium (a channel, a wire). A machine is a subsphere that embodies the flow; it itself has no subspheres. Control of the movement of things (e.g., an interruption or break) is embedded in the stages.

FM also utilizes the notion of triggering. Triggering is the activation of a flow, denoted in FM diagrams by dashed arrow. It is a dependency among flows and parts of flows. A flow is said to be triggered if it is created or activated by another flow (e.g., a flow of electicity triggers a flow of heat), or activated by another point in the flow. Triggering can also be used to initiate events such as starting up a machine (e.g., remote signal to turn on). Multiple machines captured by FM can interact by triggering events related to other machines in spheres and stages.

Note that a machine itself can be a thing that flows in another flow machine. "All matter that passes through a machine is itself a machine, itself has structure, and itself engages in its own operations... A machine is an entity that affects other entities, but that is also affected by those other entities it affects and interacts with"

Example: According to Hillier, Hanson, and Peponis [12], buildings are physical constructions, arrangements of space, and objects in a style culture. It is the organization of space that makes a building unique and sets works of architecture apart from other artifacts. A descriptive theory of space includes a level of the identification and representation of spatial elements at which boundaries are identified as a continuous boundary perforated by entrances, possibly with other boundary-entrance pairs within. Hillier, Hanson, and Peponis [12] introduce related diagrams of a simple plan and a representation of its boundary structure as a graph in which circles represent bounded spaces and lines represent relations of direct permeability (Figure 2). 

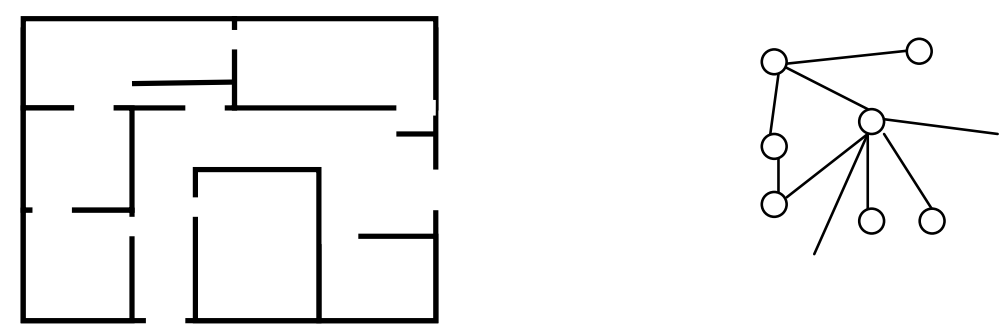

Figure 2. Left, a Simple Plan; Right, its Boundary Structure as a Graph in which Circles Represent Bounded Spaces and Lines Represent Relations of Direct Permeability (redrawn from [12])

FM representation exhibits both special and logical characteristics of these diagrams, as shown in Figure 3, a schema of the flows of things among the spaces in a house. With respect to any thing (object), the diagram shows an entry into the block of space, with possible rejection (being received includes arrval and acceptance or rejection); upon acceptance, the object is received, nay be processed (as will be shown in the next diagram of trash flow), released, and transferred to the outside. Thus, a block of space can be viewed as an arena encompassing several states of things and activities flowing through and occurring in that space.

Typical physical maps use space as a starting position: space exists by itself, furnished with things (e.g., a collection of sensors that provide data). This kind of map is a purely static relational scheme used to construct the totality of the situation and point to where other details can be added. By contrast, in FM, an empty space embodies a synchronic order of states (stäges of creation, release, transfer, receiving, and processing of thing in the space Space in FM has dynamic content. Activities (stages) and their connections (flows) in space establish a flow system (machine) representation.

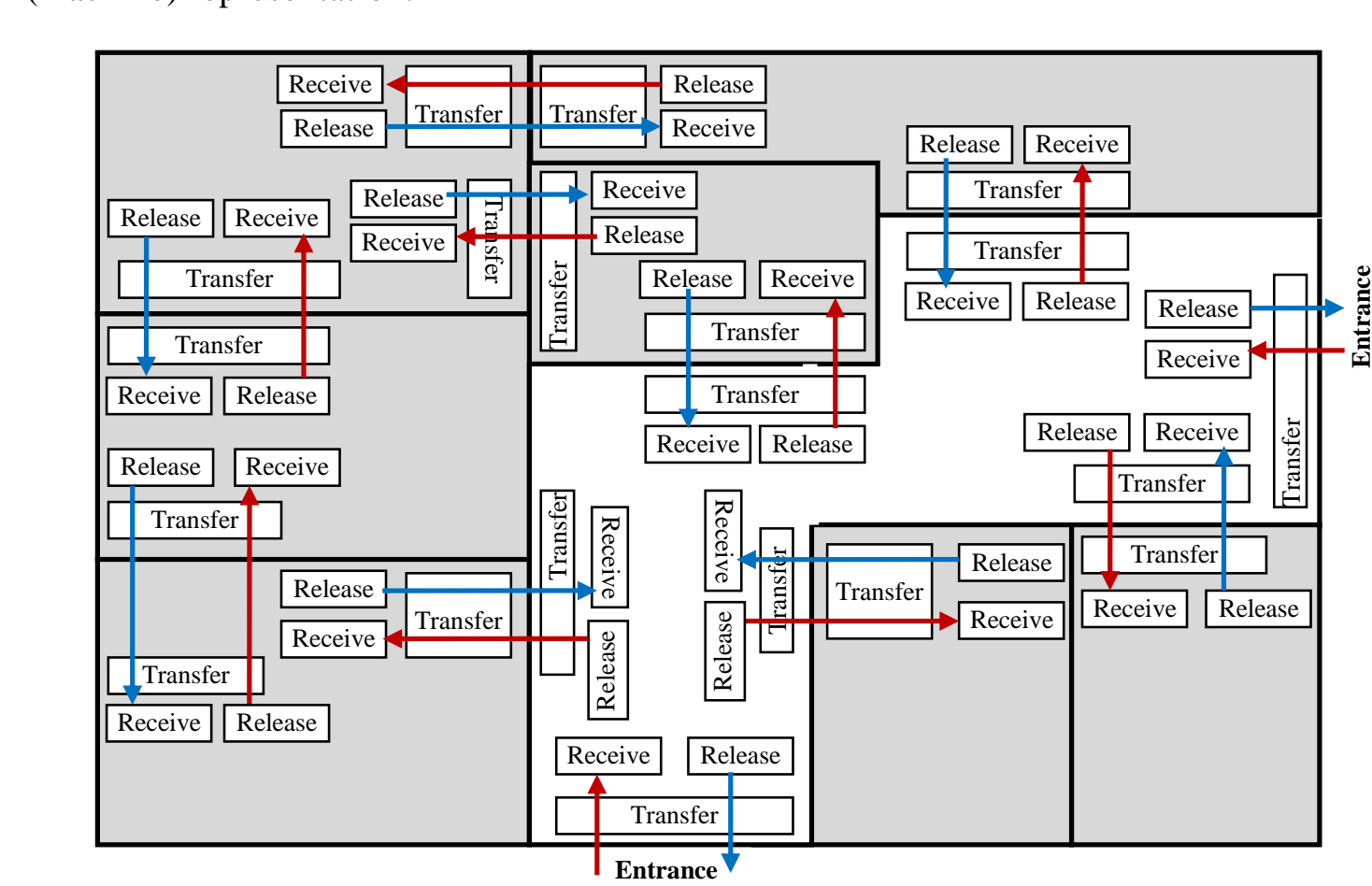

Figure 3. FM Representation of the House Blueprint in the Example 
Figure 4 shows the flow of trash through the house. Trash is created in every block of space (circles 1 through 8) for collection in a receptacle (9) in the upper left block. The arrows show the flow of trash to this storage receptacle, ignoring multiple paths. At a certain time, the stored trash is compressed (processed, 10) and taken (bold arrows) outside the house (11). Similar schemata can be drawn for all types of flows, including those of people, electricity, and conditioned air.

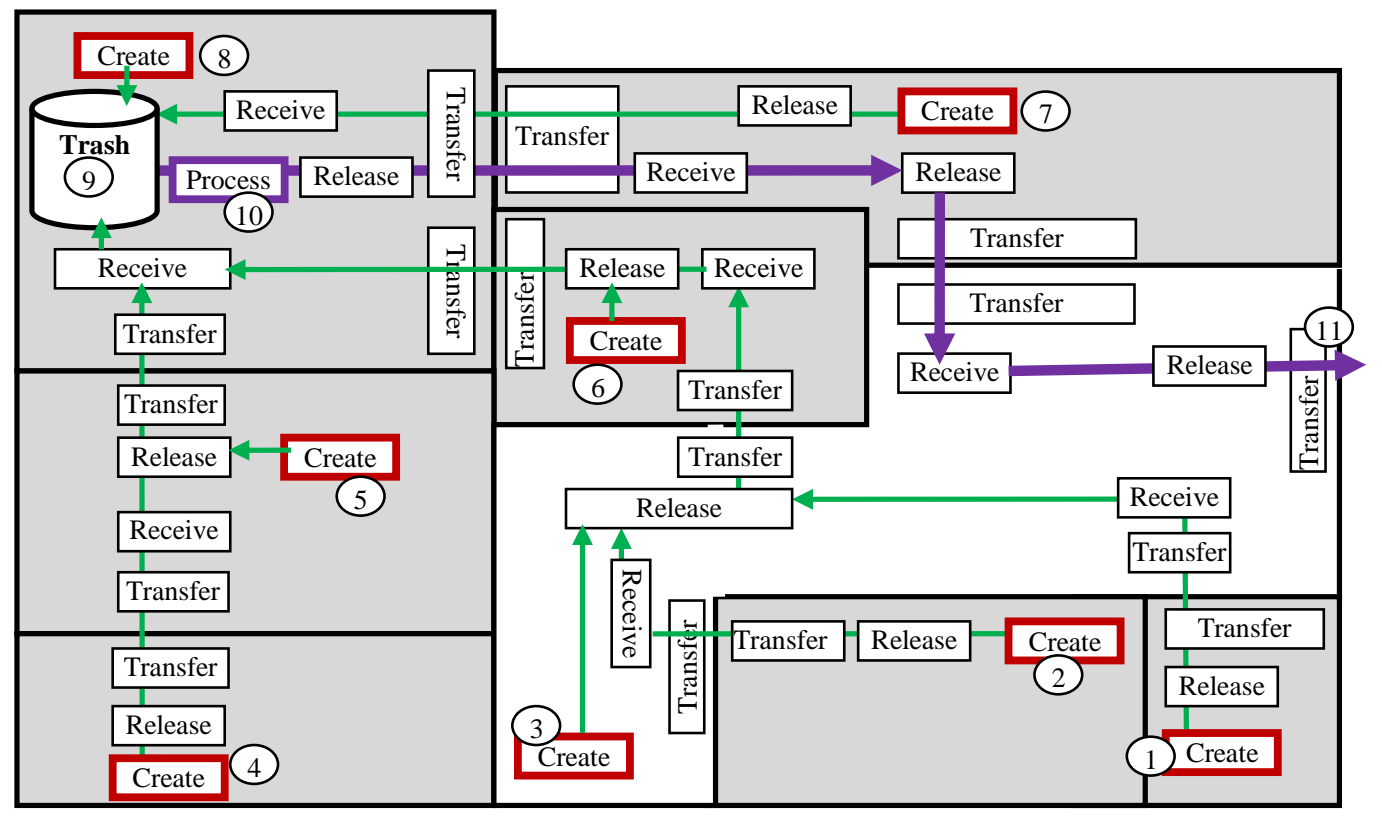

Figure 4. FM Repesentation of Flow of Trash

\section{Positioning of Sensors}

Ressouche, Tigti and Carrillo I13] studied the design of an application for use in elderly health care to monitor a person's activity in an instrumented home. The instrumented home, described liagrammatically in Figure 5, has an array of sensors to locate the person and Cetermine whether the person is standing, sitting, lying, etc. The goals are recognition of different activities and sending of several kinds of alarms depending onsensor observation results.

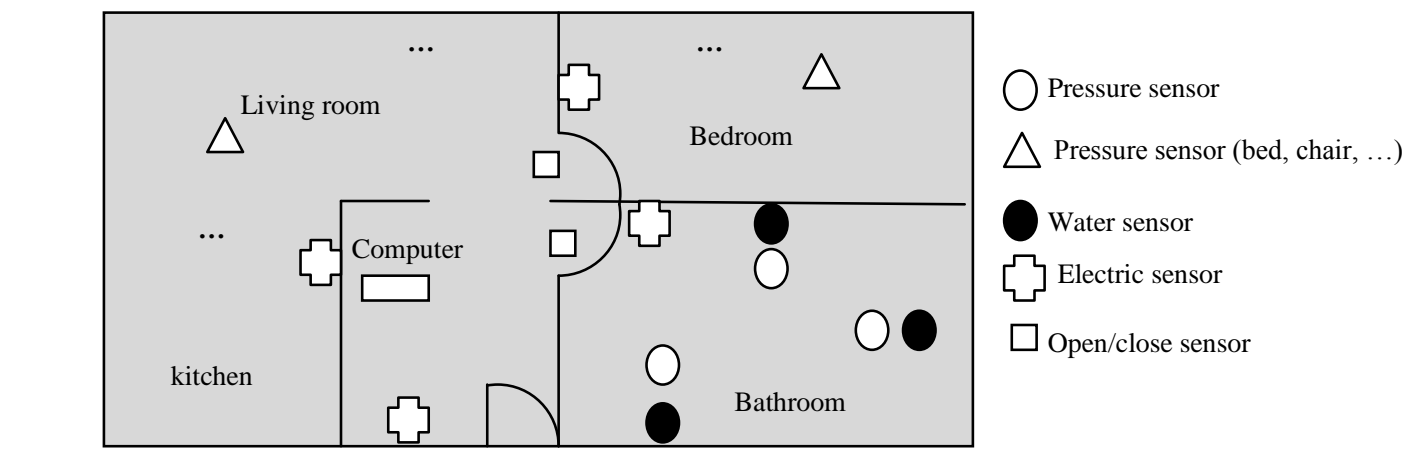

Figure 5. General View of Instrumented Home for the Elderly (Redrawn, Partial from [13]) 
In this description, sensor locations are determined by examining the architectural sketch of the home and the locations of equipment and furniture. Such positioning takes each internal flow as a black box; thus, it does not distinguish among stages of progress in a given block of space. Consider the following sample problems:

- Sensors are assigned to doors to check whether they are open or closed, in addition to pressure sensors set to recognize an entering/exiting person (Figure 5). This arrangement fails to distinguish between transferring and receiving. For example, a person can stand by the bathroom door to enter the bathroom, open the door, then, upon changing his/her mind, close the door. Accordingly, the sensors indicate transfer of the person from the living room to the bathroom, but the person is still in the living room. The designer has failed to locate another pressure sensor on the other side of the door (inside the bathroom) to indicate that the bathroom has received the person. We assume here that no sensors are used on the body of the person.

- Suppose that a person has entered the bathroom and turns on the shover then waits for the water to get warmer. Suppose that he or she suddenly has a heart attack and falls unconscious. For a period of time the system wil consider the person to be taking a shower while in actuality he or she is not in the shower area. In Figure 6, the designer failed to assign arpressure sensor in this area.

In Figure 5, it is possible that Ressouche, Tigli, and Carrillo [13] ignored these locations and types of sensors because they were not required by users; nevertheless, there is no conscious conceptualization of independent "spheres" in the topology of the space, or of internal phases of morement progression in any particular conceptual block space. For example, in the bathroom, the shower is an independent subsphere even througheno walls separate it from other areas of the bathroom. The shower is a machine that transfers in, receives, processes, releases, and transfers out a person. Each of these discrete phases may need to be explicitly recognized as a separate activity.

Figure 6 shows the drawing of this elderly nome as a machine. The stages of flow in the living room sphere are not enclosed in a box since this sphere forms the central portion of the housep. Note that, if we wish, it can be divided into several subspheres, e.g., entrance area, centrat area, window area, etc. Thus, the living room is a place in the house where a person is transferred in/out and received/released (circles 1 and 2). Suppose the person goes toward the bathroom (3). The two black circles indicate the distinction between transfering in (e. Standing on the door sill) and receiving (actually entering). A third sensor can be installed just outside the door to indicate the stage of transfer outside the home sphere.

Figure 7 shows the progress of the flow of that person inside the house. He goes to the bathroom (3), where he is received (4), proceeds to the sink (5 and 6), then leaves the sink (7) and the bathroom (8) to return to the living room (9). There he goes to the sofa (10) to be received (11), where he sits (12). He then turns on the TV (not shown; black circle on dashed arrow (13) denotes sensing of this activity). This creates a new thing (14) inside the house, the viewer who is being processed (15 - entertained). Thus, two conceptual things exist concurrently: the physical person and the viewer, two simultaneous roles of the same person. The person could now use the telephone, thus triggering (16) the creation of a phone caller (17).

The path of these activities is mined with sensors of appropriate types. Positioning of these sensors is based on a schema of the house machine, just as, say, the flow of its electricity is embedded with circuit breakers, fuses, amplifiers, etc. Some of these possible positions of sensors may not include actual sensors because they are not needed, or spatial boundaries may not be clear; nevertheless, such decisions are taken into consideration by the designer. 


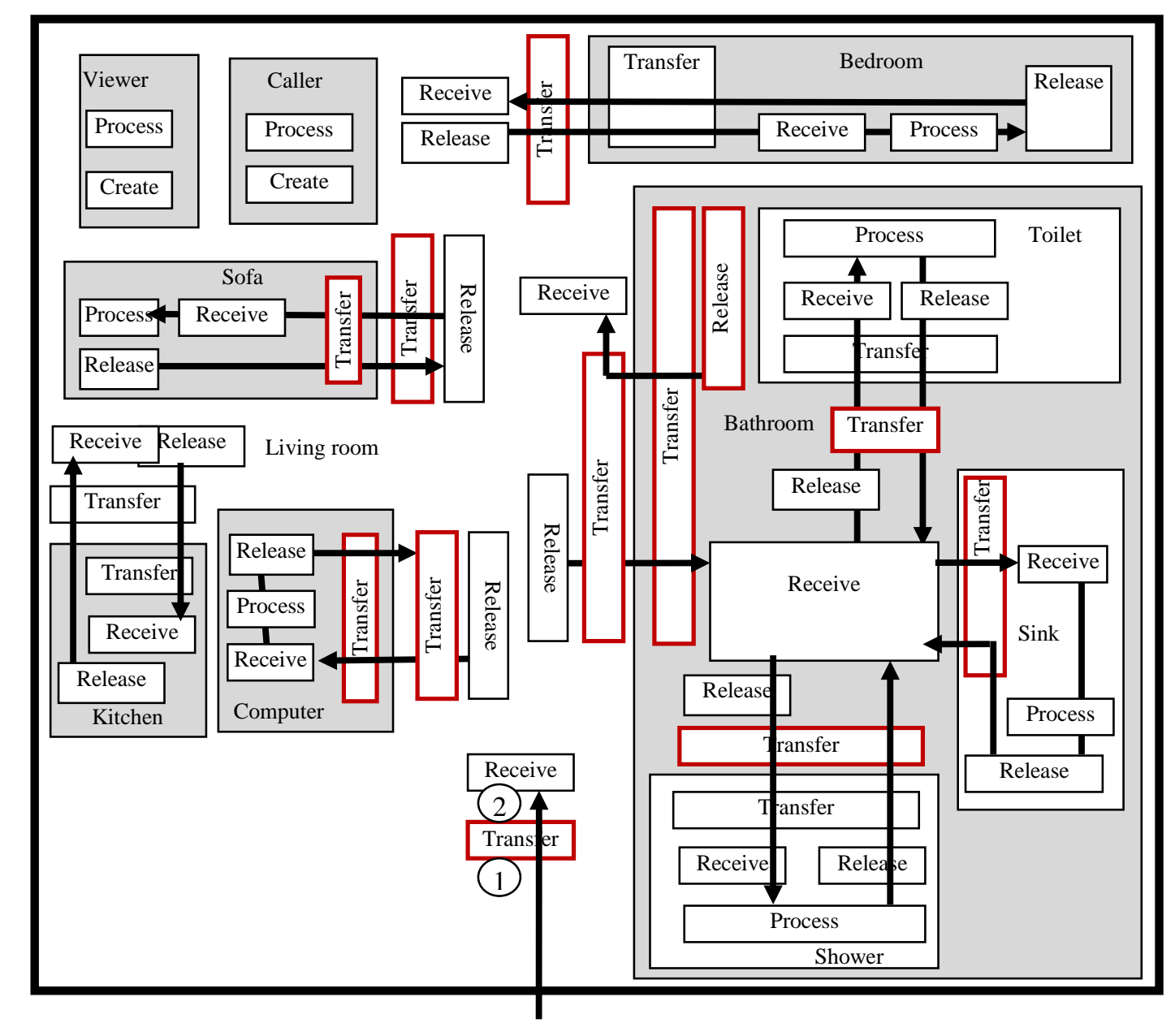

Figure 6. FiM Representation of the House

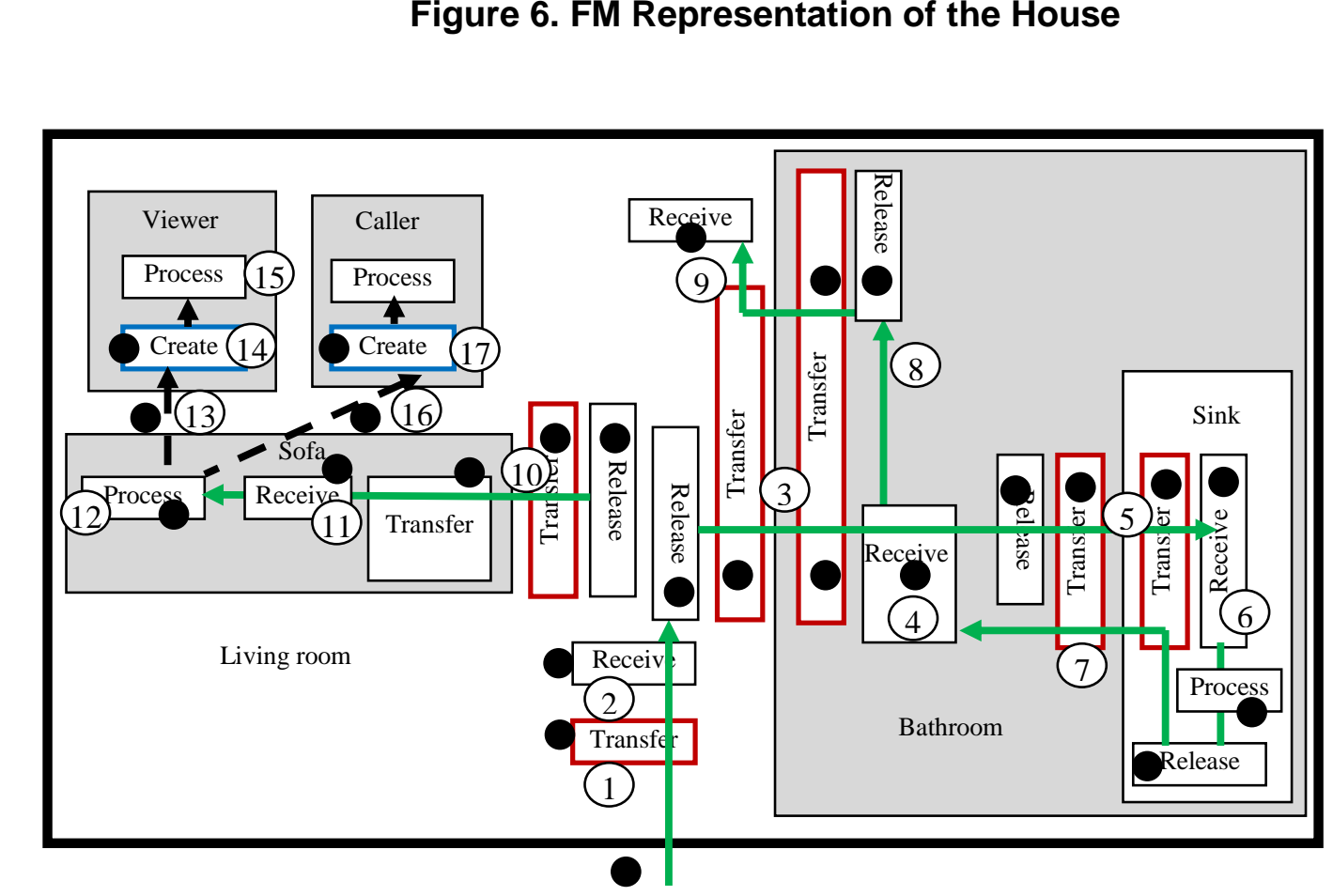

Figure 7. Sample Progression of Activities 


\section{Classifying Activities}

Smart homes equipped with monitoring devices ensure a safer environment and more effective caring through monitoring of life activities such as bathing, dressing, and cooking. The data collected have many applications in areas such as security, health, and emergency.

Extensive research has been conducted on sensor-based recognition of activity types, with most of the work linking the type of activity to the type of sensor used, such as:

- Movement activities monitored by movement detectors usually mounted in ceilings

- Contact activities using contact switches installed on doors, fridge, microwave, etc.

- Location activities using pressure mats installed on chairs, sofas, beds, sink front, etc.

Context has also been used in recognizing activities, including the room where the person is located (shower area) and interactions with objects (turning on the TV). Activities are also related to other activities, e.g., grooming can comprise, related activities such as washing and combing hair, and preparing a drink courd be divided into activities such as preparing cold or hot drinks and moving around and interacting with objects [14]. For example, preparing a cold drink con ists of going to the cupboard and taking out a cup, going to the fridge, bpening the fridge, taking out the juice, and pouring the juice into the cup ofmeral, actiyities can be recognized by interactions with objects in the enviroment and by the movements of persons within the environment [15].

To represent these sequences and structures of activities, it is beneficial to design an ontology of activities [14]. Accordingy, Hong et@b [14] designed such an ontology:

The interaction with objects andmoyements involved in an activity are recorded by associated sensors which send signals to the central management system for processing. The interrelationships between sensors, contexts and activities can be represented by a hierarchical hetwork of ontologies. [14] (Italics added)

In such an approach activities are grouped according to the room where they are performed; hence, hierarchical interrelationships are constructed among sensors, related contexts, and activities within a room location. In the example shown in Figure 8, a semsor is represented by an oval-shaped node, and rectangular nodes represent activities and objects.

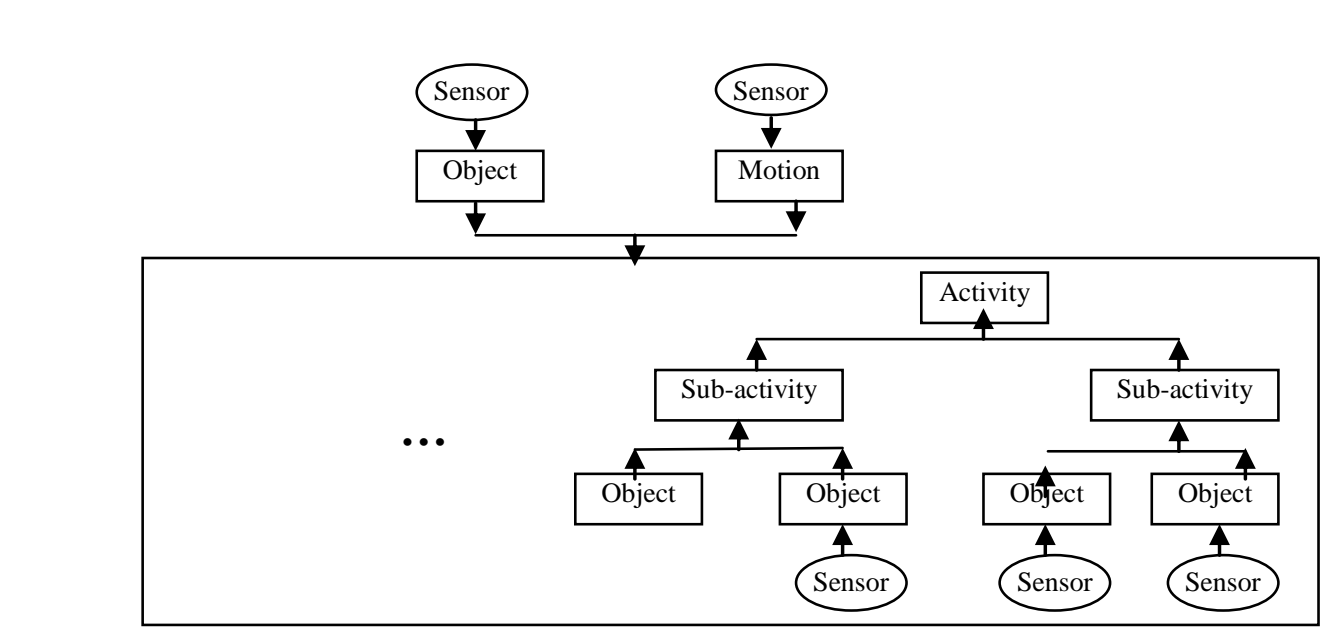

Figure 8. An Ontology of Activities

(Partial; Redrawn from [14]) 
For example, the kitchen door sensor and the related motion sensor indicate the activity of making a drink, which can be a hot or cold drink, which in turn can be juice or milk from the fridge, as indicated by its sensor (see Figure 9). "Sensors, once activated, present contextual evidence such as which room the inhabitant is in, which objects the inhabitant is interacting with and whether or not an inhabitant is moving around the home or room. All of this information provides valuable evidence which in turn can be considered indicative as to what activities the inhabitant is performing" [14].

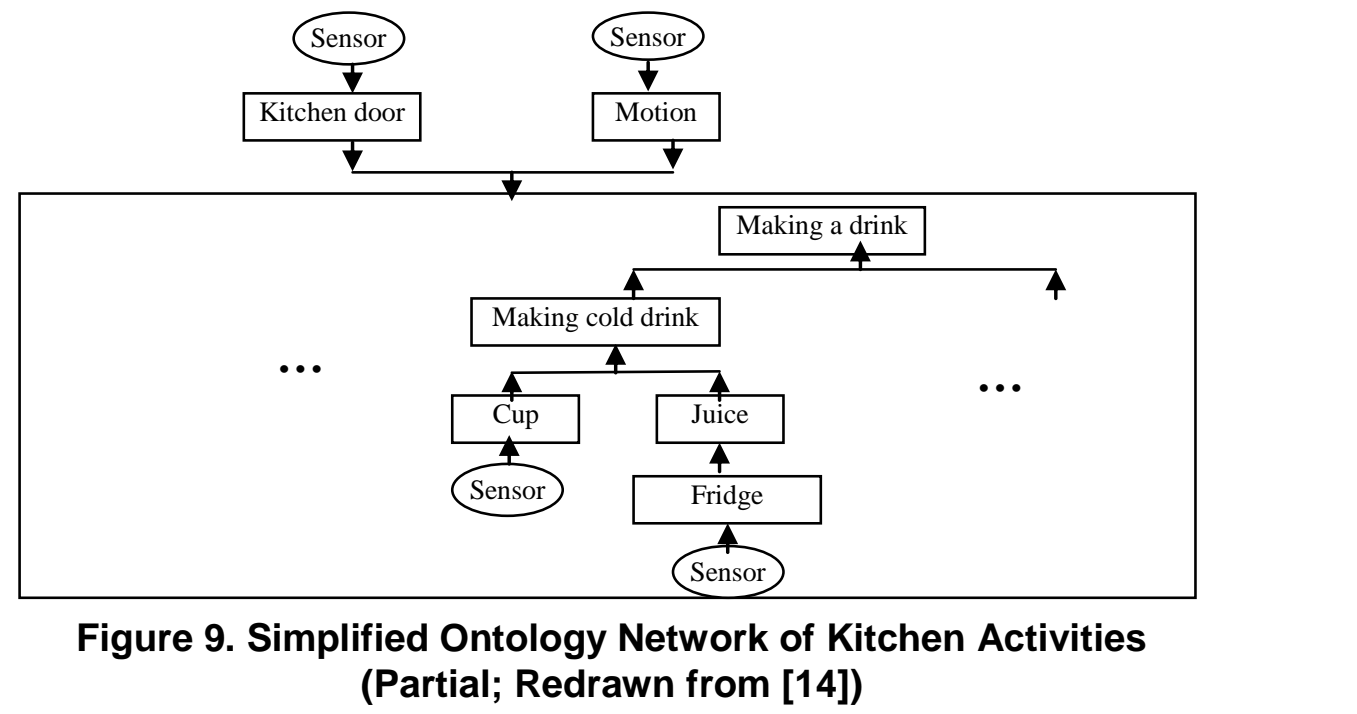

But such an ontology of activities lacks generic classification since it is based on physical devices to recognize activities in the real world. Ontology may mean providing a classification of entities and elationships in all domains or spheres of being (e.g., Porphyrian Tree: thing, material/immaterial, animate/non-animate, ...). Ontology may also be understood as domain ontology, a structured, taxonomic representation of the entities and relations existing within a particular domain of reality.

Since the formal languages and associated reasoning systems available for use with ontologies are stillfalrly limited in their capacities (see, for example, Luger, 2008 [17]), the use of ontologies to bring about regimentation of the available information requires a maximum amount of clarity and precision at each step in the process of Constructing a domain ontology. [16] (Italics added)

FM can furnish a foundation for generic classification of activities. In FM, a house is a machine constructed from spheres and submachines. A machine is the place where activities are realized. Thus, an activity means that a thing flows in the machine, in a state of being created, released, transferred, received, or processed, or a mixture of these activities that may form chains of flow. Creation, release, transfer, receiving, and processing provide basic categorization of activities. It can be observed that chains of activities can be categorized as follows:

- Movement activity of release, transfer, and receiving, involving the movement of things through stages or machines.

- Change activity that involves creation and processing. Thus, in the example of a home with close monitoring (Figure 7). turning on the TV creates a viewer that is processing (e.g., being entertained). Create here indicates the appearance of a new state of activity in the house machine that does not involve movement. 
Note that in FM conceptualization, movement as a term used in the smart home literature is not an activity; rather, it is a flowthing. Suppose that in the TV viewer example above, we want to monitor the person's hand. As shown in Figure 10, a movement is created and processed (1) to trigger (2) movement of the TV control button (3), in turn triggering the creation and transmitting of a signal to the TV (4). Also, movement can be released, transmitted, and received, as in the case of mechanical wheels.

Hence, Figure 11 shows the classification of activities according to FM conceptualization.

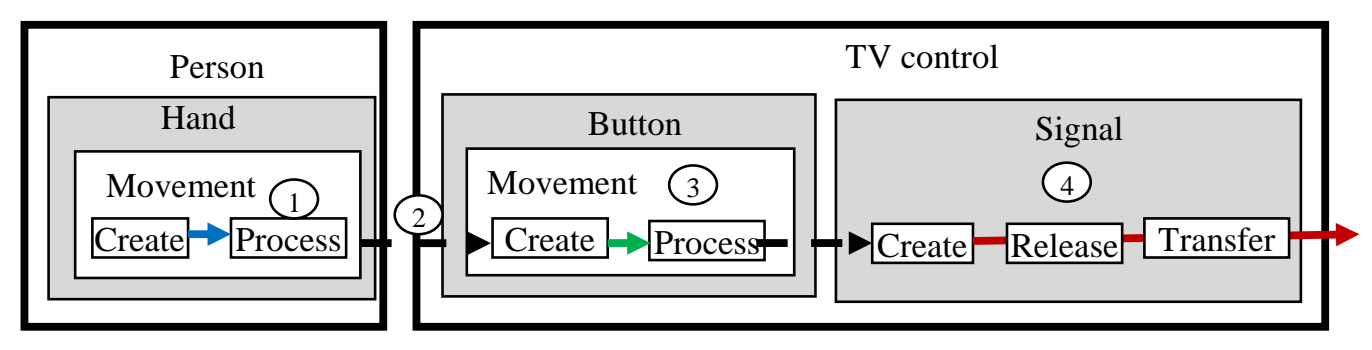

Figure 10. Hand Movement is a Thing that Flows through the Activities of Create and Process

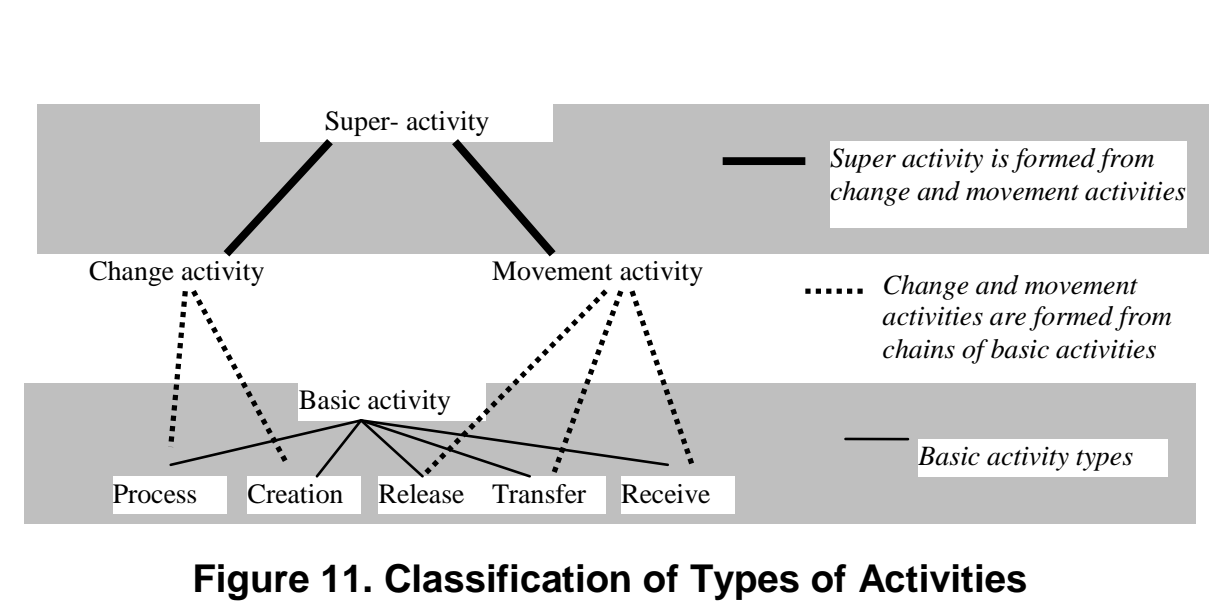

Such a classification can be used in deciding the types of activities to be monitored. For xample, in the previously mentioned case of the activity of making a drink (Figure 9) given by Hong et al. [14], Figure 12 shows what is called an evidential network of drinking activity recognition. We assume that a person who is sitting on the sofa, moves to the kitchen ( 1 and 2 ). This is detected by his/her transfer from the sofa (1) and then opening of the kitchen door (2). To further exhibit the features of FM in terms of time representation (used in the next section), Figure 12 shows a sequence of activities in which slots of time are created and consumed (surrounding box). It is also assumed that only a single person is in the house; thus, the one who has left the sofa is the same one who opens the kitchen door. Accordingly, the sensors report entering the kitchen (3), opening the fridge door (4), taking the milk container (5) that flows to the person's hand (6) and mouth (7). (Here we ignore the details of the milk container.) 


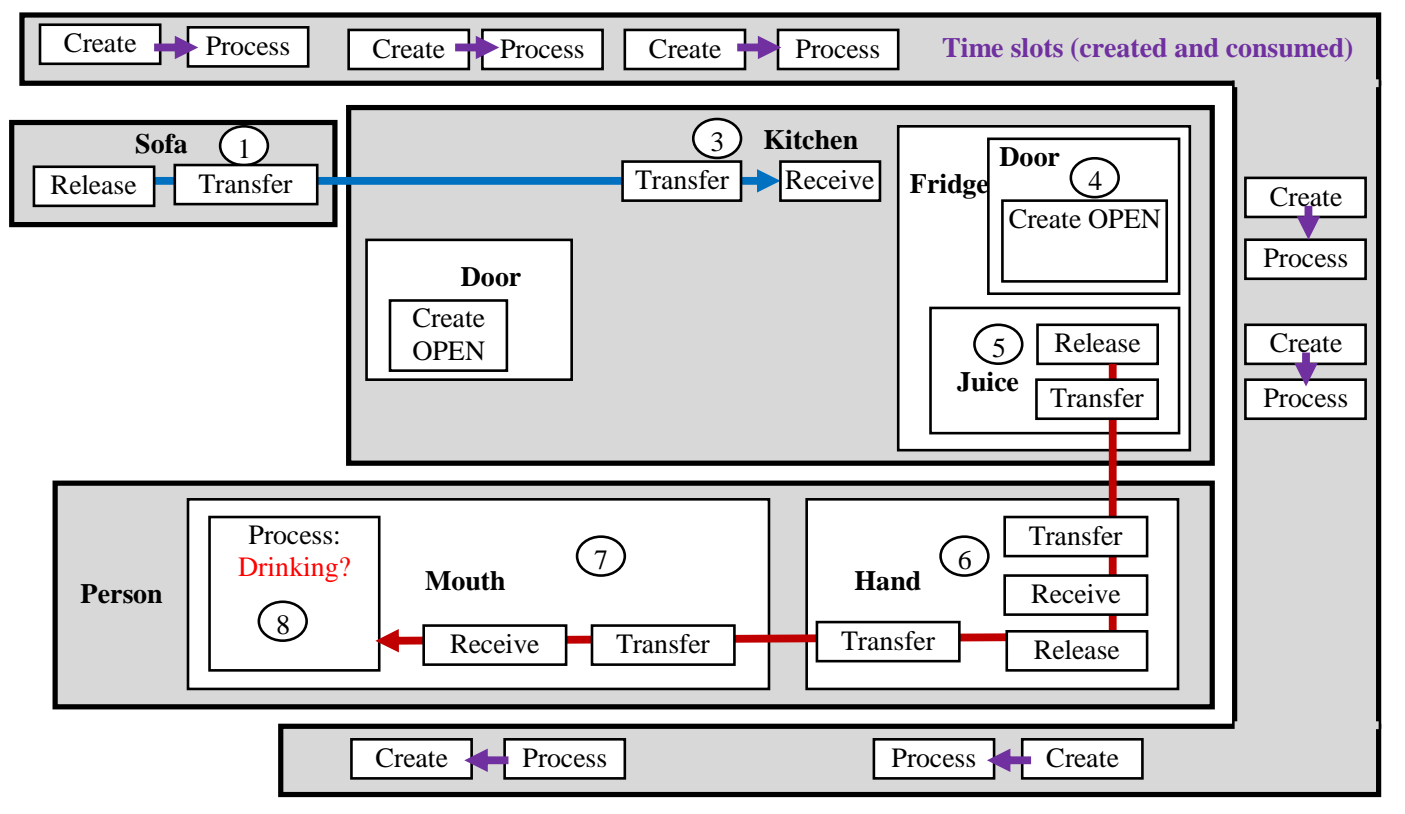

Figure 12. Description of Activities that Lead to Drinking

This flow-based description of activities is simllar to a workflow of activities; it draws a conceptual topology of movement of person an Gaice and changes in states of doors in the contexts of person and fridge. In such a representation, we can see clarity and precision at each step of activity monitoring. This precision is in clear contrast to Figure 9, the simplified ontology network of kitchen activities given by Hong et al. [14], which is static coes not reflect the continuity of events, and lacks pure conceptualization by incorporating,such terms as sensors.

Accordingly, an ontology of activitios can be based on a structure (e.g., a hierarchy) that is supplemented by the dynamic flow of things. Such a research approach could incorporate static relationships of machines.

\section{Activity Inference}

Additionally, Hong eal. [14] propose evidential networks of context-aware activity inference in which lower-level activities are used as evidence of higherlevel activities. They use notions such as noise and sensing errors and also the Dempster-Shafer theory of evidence [18]. The general process involves selecting alternatives in their hierarchal ontology of activities to maximize the probability of the occurrence of a certain activity.

Similar ontology-based activity recognition was used by Chen and Nugent [19] in modeling sensors, objects, and activities to exploit logical semantic reasoning in developing an incremental process for activity recognition. Their conceptual activity model includes activity with its various relations to time, location, duration, goals, resources, etc. Their ontology consists of an activity hierarchy in which each node denotes a type of activity that is described with a number of properties.

The scenario runs as follows: In first instance the kitchen door sensor is activated. Then the system detects the activation of the sensors attached to a cup, a teabag container and a milk bottle in a temporal sequence. Suppose no other sensors were activated during this period that the above sensors become active. The question is therefore which ADL [activity of daily living] just took place? [19] 
FM presents a potential new approach to inferences. A knowledge base of activities can be constructed for comparison with data given by the sensors. For example, make hot tea can be represented as in Figure 13, where it is assumed that the cup (1), teabags (2), and hot water (3) can be found only in the kitchen (4). Accordingly, when

- The cup is taken from its compartment (5), this takes a time period t (6).

- The teabag is taken from its place (7), this takes a certain time period (8).

- The hot water is brought in (9), this takes a time period that occurs after $t$ (10),

The teabag and hot water are then mixed (11) in the cup to make hot tea (12). Note the constraint that receiving the hot water occurs only after receiving the cup. Note also that the so-called contextual information of the kitchen as a location is an integral part of the FM diagram.

Now, suppose that sensors received data indicating the activity shown in Figure 14, as follows:

- The kitchen door has been opened (1), and

- Someone has entered the kitchen (2)

- The cup has been taken from its compartment

- Hot water has been poured out

- A teabag has been taken

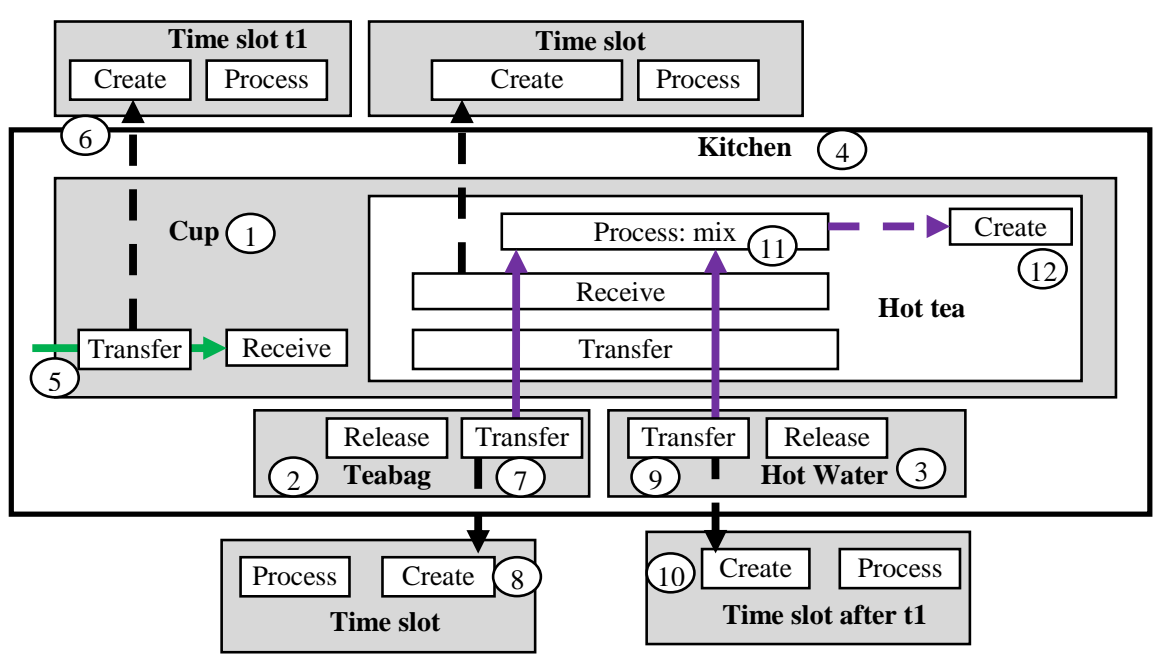

Figure 13. Make Drink Activity

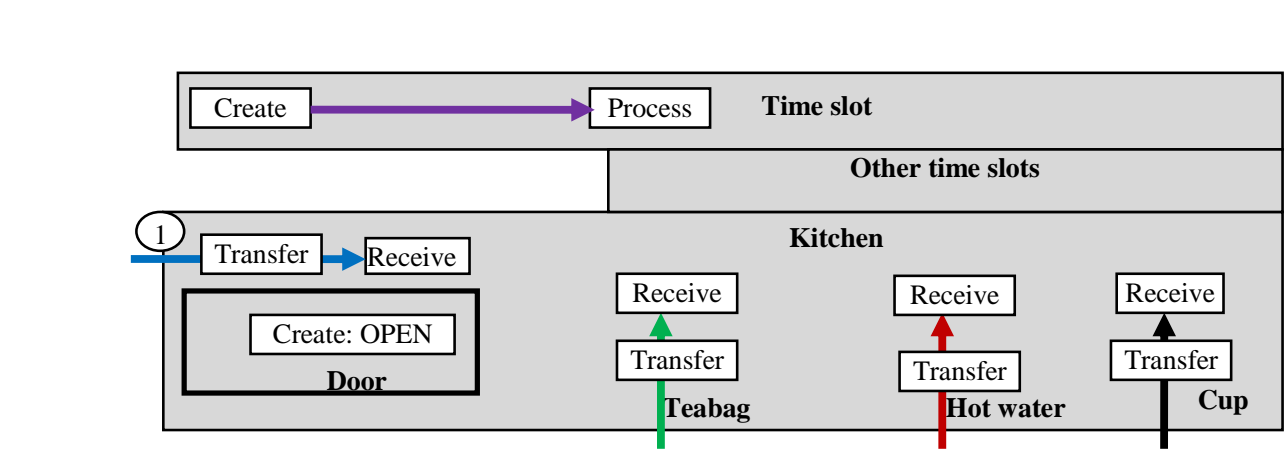

Figure 14. Actual Data Received from the Sensors (for Simplicity, Some Boxes are Omitted) 
Then, consulting the knowledge base of Figure 13 and comparing the two diagrams, it can be concluded that the activity of making tea has occurred. Note that in addition to the constraints that the cup is taken first, then the hot water is poured (Figure 13), there is the constraint in Figure 14 that the door be open and someone enter the kitchen before any of the other activities can take place.

The issue of how to integrate activities and how to conduct the search for a certain subdiagram in the knowledge base is a research issue beyond the scope of this paper.

\section{Conclusion}

This paper contributes to the notion of "house as machine" by proposing a methodology for producing engineering-type diagrams of machines that can be applied to smart home design in resolving such issues as where to position sensors, how to classify, activities, and how to recognize activity. It is clear from the given examples that FM presents a new methodology for representing activities in smart homes while addressing related issues such as inferences for recognizing activities. This new approach is of course in need of more research to resolve issues raised in the papef such as development of an ontology that embeds flows, and comparisons of FM graph are also needed

\section{References}

[1] Le Corbusier, "Towards a New Architecture (Vers une architecture)" translated by F. Etchells from the original 1923 French edition, Architectural Press, (1927); Version used:1970 Paperback of 1946 edition, p. 89.

[2] M. Storper, "The City: Center of Economic Reflexivity, A Chapter in The Regional World", Guilford, London and New York, (1996).

[3] B. Hillier and J. Hanson, "The Social Lôgic of Space", Cambridge University Press, Cambridge, (1984)

[4] Wittgenstein, "What Is a Machine?" no. 193 in Philosophical Investigations (1953) .

[5] G. Deleuze and F. Guattari, "Anti-Qedipus: Capitalism and Schizophrenia”, University of Minnesota Press, Minneapolis, (1983).

[6] L. R. Bryant, "Towards a Machine-oriented Aesthetics: On the Power of Art", at The Matter of Contradiction Conference, Rínousin, France, (2012).

[7] S. Al-Fedaghi, "Heraditean Ontology for Specifying Systems", International Review on Computers and Software (IRECOS), vol 10, no. 6, (2015). http://dx.doi.org/10.15866/irecos.v10i6.6493

[8] S. Al-Fedaghi and N. Aljallal, "Conceptual Schematization of Microcontroller and Assembly Language", International Journal of Software Engineering and Its Applications, vol. 8, no. 10, (2014), pp. 179-190.

[9] Al-Fedaghi, "Schematizing Proofs based on Flow of Truth Values in Logic", IEEE International Conference on Systems, Man, and Cybernetics, Manchester, UK, (2013), pp. 194-200.

[10] S. Al-Fedaghi and F. Al-Kanderi, "Integrating Security Concerns into Software Development", International Journal of Security and Its Applications, vol. 7, no. 3, (2013), pp. 235-248.

[11] S. Al-Fedaghi, 'Developing Web Applications", International Journal of Software Engineering and Its Applications, ol. 5, no. 2, (2011), pp. 57-68..

[12] B. Hillier J. Hanson, and J. Peponis, "What Do We Mean by Building Function?", pp. 61-72 in Designing for Building Utilisation, Edited by J. Powell. Spon, London, (1984).

[13] A. Ressouche, J.-Y. Tigli and O. Carrillo, Software Composition: 10th International Conference, SC 2011 (2011), pp. 165-180.

X Hong, C. Nugent, M. Mulvenna, S. McClean, B. Scotney, and S. Devlin, "Evidential Fusion of Sensor Data for Activity Recognition in Smart Homes", Pervasive and Mobile Computing, vol. 5, (2009), pp. 236-252.

[15] M. Philipose, K. P. Fishkin, M. P. Patterson, D. Fox, H. Kautz and D. Hahnel, "Inferring Activities from Interactions with Objects", IEEE Pervasive Computing, vol. 3, no. 4, (2004), pp. 50-57.

[16] R. Arp, "Ontology: Not Just for Philosophers Anymore”, Practical Philosophy, vol. 10, no. 1, (2010).

[17] G. Luger, Artificial Intelligence: Structures and Strategies for Complex Problem Solving. AddisonWesley, New York, (2008).

[18] A. P. Dempster, "A Generalization of Bayesian Inference", Journal of the Royal Statistical Society (Series B), vol. 30, (1968), pp. 205-247.

[19] L. Chen and C. Nugent, "Ontology-based Activity Recognition in Intelligent Pervasive Environments", International Journal of Web Information Systems, vol. 5, no. 4, (2009), pp. 410-430. 


\section{Author}

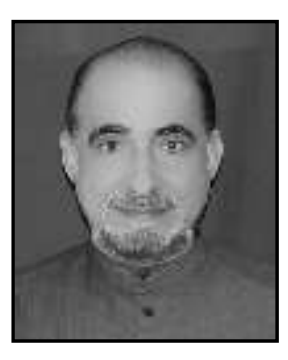

Sabah Al-Fedaghi holds an MS and a $\mathrm{PhD}$ in computer science from the Department of Electrical Engineering and Computer Science, Northwestern University, Evanston, Illinois, and a BS in Engineering Science from Arizona State University, Tempe. He has published two books and more than 250 papers in journals and conferences on software engineering, database systems, information systems, computer/ information privacy, security and assurance, information warfare, and conceptual modeling. He is an associate professor in the Computer Engineering Department, Kuwait University. He previously worked as a programmer at the Kuwait Oil Company, where he also headed the Electrical and Computer Engineering Department (1991-1994) and the Computer Engineering Department (2000-2007).

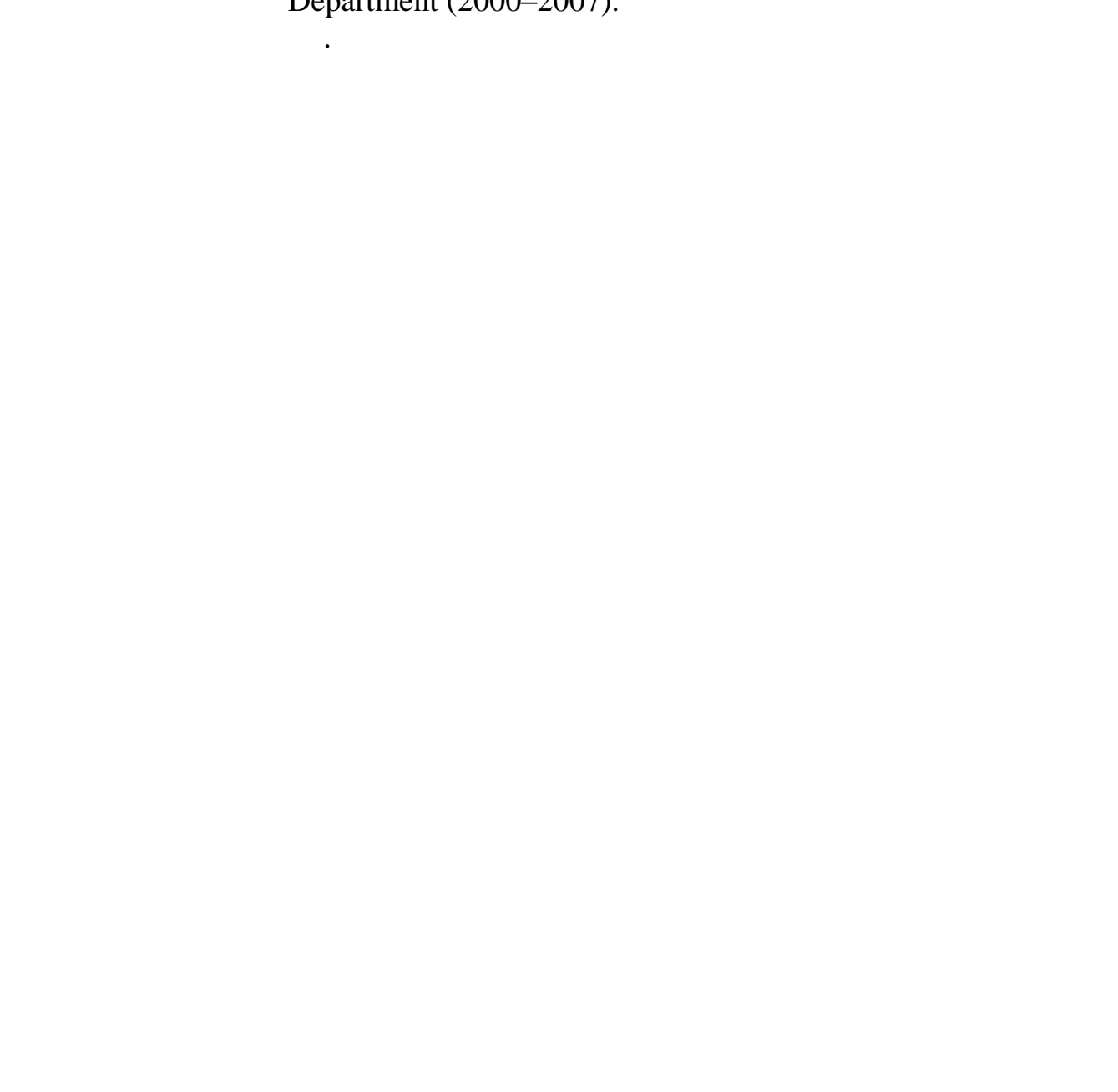

